\title{
CREATINAFOSFOQUINASE-ISOENZIMA MB MASSA (CK-MB MASSA) E TROPONINA I (cTnI) EM CÃES (Canis familiaris)
}

\section{CREATINE PHOSPHOKINASE ISOENZYME-MB MASS (CK-MB MASS) AND TROPONIN I (cTnI) IN DOGS (Canis familiaris)}

\author{
Millena Vidal Freitas ${ }^{1^{*}}$ \\ Felipp da Silveira Ferreira ${ }^{2}$ \\ Flavia Lopes Barretto ${ }^{3}$ \\ Elisabete Sales Corrêa ${ }^{4}$ \\ Claudio Baptista Carvalho ${ }^{4}$ \\ ${ }^{1}$ Clínica Veterinária Vida Vet. Belo Horizonte, MG, Brasil \\ 2Universidade Vila Velha, Vila Velha, ES, Brasil \\ ${ }^{3}$ Médica Veterinária Autônoma. Rio de Janeiro, RJ, Brasil \\ ${ }^{4}$ Universidade Estadual do Norte Fluminense Darcy Ribeiro, Campos dos Goytacazes, RJ, Brasil \\ *Autor para correspondência - millevidal@gmail.com
}

\section{Resumo}

Os marcadores cardíacos, sobretudo a CK-MB massa e a cTnI, tem demonstrado um papel essencial seja em cardiologia clínica ou cirúrgica veterinária e humana, permitindo um diagnóstico mais preciso e acurado nas lesões miocárdicas. O objetivo deste trabalho foi mensurar os marcadores cardíacos em tela na Medicina Veterinária, aprimorar sua utilização e obter informações sobre estes métodos laboratoriais não invasivos que permitem o monitoramento da integridade da célula miocárdica. A quantificação dos parâmetros foi feita do soro de cães saudáveis atendidos na rotina do setor de Clínica Médica de Pequenos Animais do Hospital Veterinário da Universidade Estadual do Norte Fluminense Darcy Ribeiro. O kit de ensaio imunométrico por quimioluminescência turbo (IMMULITE Turbo, Siemens®) humano mostrou-se eficaz na avaliação na espécie canina, tanto para CK-MB massa quanto cTnI. Além disso, os valores encontrados para CK-MB massa e cTnI contribuirão significativamente para a rotina clínica ou para os trabalhos experimentais em cães.

Palavras-chave: CK-MB massa; cTnI; lesão miocárdica; marcadores cardíacos.

\begin{abstract}
Cardiac markers, especially CK-MB mass and cTnI, have an essential role in both human and veterinary clinical and surgical cardiology, allowing a more precise and accurate diagnosis in myocardial lesions. The goal of this work was to measure these cardiac markers in veterinary medicine, improve their use and provide information about these laboratory methods that allow non-invasive health monitoring of the myocardial cell. Parameters quantification was obtained from sera of healthy dogs examined during routine procedures at the Small Animal Veterinary Hospital of Universidade Estadual do Norte Fluminense Darcy Ribeiro. The human chemiluminescence
\end{abstract}


essay turbo kit (IMMULITE Turbo, Siemens ${ }^{\circledR}$ ) test proved to be effective in canine species for both CK-MB mass and cTnI. In addition, the values found for CK-MB will significantly contribute to clinical routine or experimental work with dogs.

Keywords: cardiac markers; CK-MB mass; cTnI; myocardial injury.

Recebido em: 12 março 2012

Aceito em: 13 março 2015

\section{Introdução}

Os marcadores cardíacos têm sido empregados rotineiramente para a detecção de enfermidades miocárdicas em medicina, sobretudo para o diagnostico de infartos agudos do miocárdio (IAM) ${ }^{(1)}$. De acordo com Santos et al. ${ }^{(2)}$ e Freitas et al. ${ }^{(3)}$, alguns dos marcadores empregados para este fim são a creatinofosfo-quinase isoenzima MB (CK-MB) e as troponinas, sendo estas mais específicas que as primeiras para os eventos de hipóxia de miocárdio.

Segundo Lopes et al. ${ }^{(4)}$ e Freitas et al. ${ }^{(3)}$, a creatinoquinase (CK) é uma molécula constituída por duas subunidades, $\mathrm{M}$ e B. Quando ocorre necrose do miocárdio, há liberação da isoenzima CK-MB para o meio extracelular e sua dosagem representa um recurso importante para a detecção de lesão cardíaca $^{(5)}$.

Em humanos, a dosagem da CK-MB vem sendo utilizada como principal método para confirmação ou exclusão do infarto agudo do miocárdio (IAM) e picos de CK-MB podem prever eventos cardíacos desfavoráveis em populações de alto risco. Entretanto, CK e CK-MB são pobres marcadores de danos no miocárdio em cães, sendo a CK-MB um marcador cardíaco menos específico que a troponina cardíaca para esta espécie. Em medicina veterinária, tem sido utilizada para avaliar cardiotoxicidade de fármacos em protocolos quimioterápicos e de peçonhas em intoxicações experimentais ${ }^{(3-6)}$.

De acordo com Aktas et al. ${ }^{(7)}$ e Yonezawa et al. ${ }^{(8)}$, a CK-MB pode ser mensurada tanto por sua atividade sérica, por meio de testes bioquímicos (CK-MB atividade), quanto por sua concentração total (CK-MB massa), por meio de ensaios imunométricos. Enquanto a dosagem de CK-MB determina a atividade da enzima, o teste de CK-MB massa detecta sua concentração, independentemente de sua atividade, o que torna o CK-MB massa mais confiável que os testes de CK-MB atividade. Desta maneira, o CK-MB massa apresenta melhor sensibilidade analítica, pois detecta enzimas ativas e inativas.

Uma vez que a literatura veterinária é escassa em relatos de dosagem de CK-MB massa em cães, há poucos valores de referência para esta isoenzima, o que dificulta sua utilização na rotina veterinária. De acordo com a literatura, as troponinas têm recebido crescente atenção como marcadores específicos de injúria celular miocárdica em humanos e, atualmente, são consideradas como os preferíveis de injúria cardíaca em mamíferos ${ }^{(5,8,9)}$. Boswood $^{(10)}$ e Kocaturk et al. ${ }^{(1)}$ afirmam que as troponinas cardíacas são exclusivamente expressas no miocárdio e têm se mostrado mais sensíveis e específicas do que outros marcadores séricos empregados anteriormente para o diagnóstico nãoinvasivo de necrose miocárdica.

Como exposto, por esta elevada sensibilidade e especificidade, a troponina I cardíaca (cTnI) tornase importante para o diagnóstico precoce do IAM em humanos ${ }^{(1,8)}$, não sendo detectável em testes 
qualitativos em pessoas sadias ${ }^{(12)}$. Da mesma forma, Santos et al. ${ }^{(2)}$ afirmam que cães e gatos podem não apresentar cTnI na circulação, mas alguns animais podem apresentar traços, ou seja, concentração geralmente inferior a $0,1 \mathrm{ng} / \mathrm{mL}$.

Embora o infarto agudo do miocárdio seja uma causa muito rara no cão e no gato, altas concentrações de cTnI têm sido associadas a muitas outras doenças cardíacas que são clinicamente relevantes nestas espécies, como, por exemplo, doenças cardíacas congênitas e adquiridas, cardiomiopatia hipertrófica, cardiomiopatia dilatada, estenose subaórtica, degeneração mixomatosa da válvula mitral, arritmias graves, efusão pericárdica, miocardites, cardiomiopatia arritmogênica do ventrículo direito, contusão cardíaca, dentre outras ${ }^{(3,13)}$. Além disso, elevações de troponina I também têm sido associadas a doenças não cardíacas, mas que afetam o coração, como torção e dilatação vólvulo-gástrica, trauma torácico, disfunções orgânicas, toxemias, uso de drogas cardiotóxicas, neoplasias cardíacas e babesiose ${ }^{(3)}$.

Segundo Amaral et al. ${ }^{(14)}$ e Adin et al. ${ }^{(15)}$, um dos fatores limitantes para a utilização de CK-MB massa e cTnI em cães é a variabilidade dos valores de referência, obtida por metodologias e equipamentos diferentes, sendo que em alguns tais valores sequer existem ou são específicos para cada metodologia. Por esse motivo, o objetivo desta pesquisa foi mensurar os valores da creatinofosfoquinade isoenzima-MB massa (CK-MB Massa) e troponina I (cTnI) em cães saudáveis, a fim de se estabelecer seus valores de referência, a partir de um "kit" de ensaio imunométrico por quimioluminescência (IMMULITE turbo®) humano, além de validá-lo para a espécie canina.

\section{Material e Métodos}

O presente estudo foi aprovado pelo Comitê de Ética para o Uso de Animais da Universidade Estadual do Norte Fluminense Darcy Ribeiro sob registro CEUA-UENF 060.

O trabalho foi realizado utilizando-se 60 (sessenta) cães admitidos no setor de cardiologia do Hospital Veterinário da Universidade Estadual do Norte Fluminense Darcy Ribeiro para avaliação de rotina. $\mathrm{O}$ número amostral foi calculado com base em um universo de 420 casos, dos quais apenas 30\% representavam pacientes saudáveis, população para a qual calculou-se o número amostral com margem de erro de $9 \%$ e índice de confiabilidade de 95\%. Empregaram-se animais sem restrição de raça, sexo, porte e peso.

Como critérios de inclusão para este estudo, utilizaram-se animais com histórico médico normal, exame físico, eletrocardiográfico e ecocardiográfico sem evidências de doenças cardíacas congênitas ou adquiridas. Foram realizadas, ainda, avaliações hematológicas como hemograma e bioquímica sérica (ALT, FA, FA, ureia e creatinina). Foram excluídos da pesquisa animais com idade inferior a 6 meses e superior a 8 anos, bem como aqueles que apresentavam sopro cardíaco, doenças concorrentes, lesões externas, alterações eletrocardiográficas, ecocardiográficas ou hematológicas.

As amostras de sangue venoso foram colhidas em seringas de $3 \mathrm{~mL}$ conectadas a agulhas $25 \mathrm{x} 07$ $\mathrm{mm}$ por acesso à veia jugular. O sangue colhido foi repassado a frascos siliconizados sem anticoagulante. Em seguida, as amostras foram encaminhadas para o laboratório de Patologia Clínica do Hospital Veterinário da Universidade Estadual do Norte Fluminense Darcy Ribeiro e ao Laboratório Plínio Bacelar de Campos dos Goytacazes - RJ. As amostras sanguíneas foram 
centrifugadas a $1500 \mathrm{~g}$ por 5 minutos, em tempo não superior a quinze minutos após a coleta. $\mathrm{O}$ produto sobrenadante foi aliquotado em volume mínimo de $500 \mu \mathrm{L}$. Tais amostras foram acondicionadas em microtubos tipo "Eppendorf" e mantidas sob refrigeração em "freezer", à temperatura de $-20{ }^{\circ} \mathrm{C}$, em tempo não superior a 30 dias, para posterior realização de análises bioquímicas. Tanto a CK-MB massa como a Troponina I foram avaliadas por ensaio imunométrico em fase sólida de quimioluminescência em equipamento ImmuliteTurbo da marca Siemens®.

Os resultados foram analisados com programa estatístico GraphPad Prism versão 5.0 for Windows (GraphPad Software®) através dos cálculos de média, desvio-padrão, variância e intervalo de confiança.

\section{Resultados e Discussão}

Os resultados obtidos são apresentados sob forma de tabelas e gráficos. Na Tabela 1 estão descritos a média, desvio padrão (DP), variância e os intervalos de confiança (IC) dos biomarcadores cardíacos que foram analisados através do soro de cães.

Tabela 1: Valores de Média, Desvio padrão, Variância e IC dos Biomarcadores Cardíacos

\begin{tabular}{llllll}
\hline & Média & $\begin{array}{l}\text { Desvio } \\
\text { Padrão }\end{array}$ & - Variância & $\begin{array}{l}\text { Intervalo } \\
\text { Confiança }\end{array}$ \\
\cline { 2 - 5 } & $0,074 \mathrm{ng} / \mathrm{ml}$ & 0,097 & 0,009 & $0,050-0,098 \mathrm{ng} / \mathrm{ml}$ \\
Troponina I (cTnI) & $0,941 \mathrm{ng} / \mathrm{ml}$ & 1,277 & 1,630 & $0,618-1,264 \mathrm{ng} / \mathrm{ml}$ \\
\hline
\end{tabular}

Os resultados obtidos de média e de IC podem também ser observados na Figura 1.

\section{Biomarcadores}

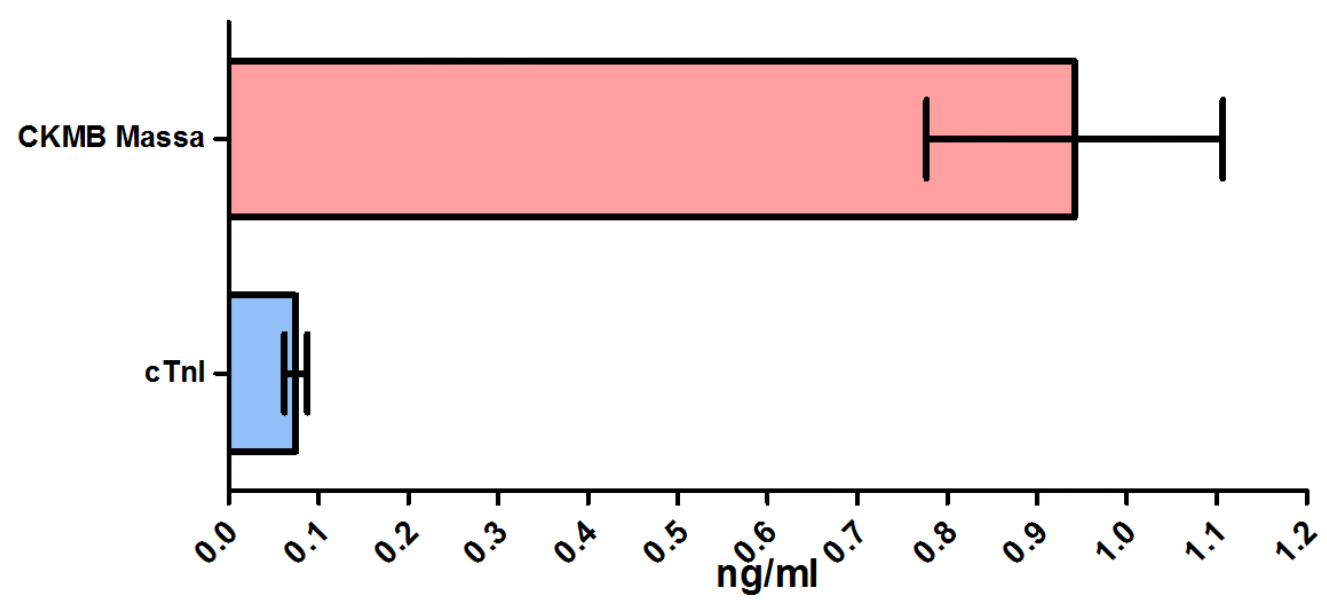

Figura 1: Média e Intervalo de Confiança dos Biomarcadores CKMB Massa e cTnI. Observa-se intervalo de confiança de $0,050-0,098 \mathrm{ng} / \mathrm{ml}$ para cTnI e de 0,618 - 1,264 para CK-MB massa. Hospital Veterinário/UENF, 2012. 
De acordo com a Figura 1, pode-se perceber que a CK-MB massa apresentou média de 0,941 $\mathrm{ng} / \mathrm{mL}$ com desvio padrão de 1,277, enquanto a cTnI apresentou um valor médio de 0,074 com desvio padrão de 0,097. De forma prática, os resultados que podem ser transferidos às rotinas clínicas e laboratoriais são apresentados em intervalos de confiança (IC) para cada um dos biomarcadores estudados, a saber:

- $\quad$ cTnI: Média: 0,074 ng/mL; IC: \pm 0,024 (0,050 - 0,098 ng/ml);

- $\quad$ CK-MB massa: Média: 0,941 ng/mL; IC: $\pm 0,323(0,618-1,264 \mathrm{ng} / \mathrm{ml})$.

A literatura médico-veterinária, ao contrário da literatura médica, ainda é escassa em trabalhos envolvendo os marcadores cardíacos; no entanto, nos últimos anos, foram publicados artigos mensurando-se as troponinas em cães normais e em cães com alterações cardíacas ${ }^{(13,16,17)}$. De acordo com a literatura, há poucos estudos com relatos da mensuração da CK-MB massa utilizandose o ensaio imunométrico quimiluminescente, Immulite®. Nos centros cardiológicos médicos, a mudança na metodologia de dosagem da CK-MB objetivou à melhoria da sensibilidade e especificidade diagnóstica e, com este avanço, passou a ser empregada na Medicina Veterinária ${ }^{(2,15)}$. Em relação à CK-MB massa, o grupo de 60 animais avaliados apresentaram média de $0,940 \mathrm{ng} / \mathrm{mL}$ e desvio-padrão de 1,277 e, por se tratar de animais em que não foram detectadas quaisquer tipos de enfermidades prévias ou concorrentes, por avaliações clínicas e complementares, sugere-se a utilização destes valores como possíveis valores de referências. Já a cTnI teve média de 0,074 $\mathrm{ng} / \mathrm{mL}$ e desvio padrão de 0,097 . Os valores encontrados são semelhantes aos encontrados por Spratt et al. ${ }^{(18)}$, Fonfara et al. ${ }^{(19)}$, Wess et al. ${ }^{(20)}$, Oyama et al. ${ }^{(21)}$ e Sleeper et al. ${ }^{(13)}$, para cTnI, embora alguns destes autores tenham se utilizado de metodologias diferentes, o que corrobora na validação dos resultados deste estudo. Tanto para cTnI quanto para CK-MB, os autores citados mensuraram os biomarcadores em animais sadios, de raça, sexo, porte e idades variadas.

Os resultados aqui observados representam que, em condições de normalidade, as amostras de cada um destes biomarcadores aferidos em cães com esta metodologia devem situar-se de acordo com os intervalos supracitados. Quando confrontados com os dados obtidos na literatura especializada, percebe-se que estes valores são sempre reduzidos e próximos, como pode-se observar na Figura 2.

Devido aos poucos estudos veterinários existentes em relação à CK-MB massa para cães, existem poucas informações disponíveis sobre os valores de referência da CK-MB massa nessa espécie; no entanto, em relação à CK-MB atividade (ou sérica), existem mais valores de referência relatados. Santos et al., (2) conseguiu estabelecer valores de CK-MB massa de normalidade para animais sadios, estando este valor compreendido de 0 a $0,54 \mathrm{ng} / \mathrm{mL}$, o que corrobora com Ferreira et al. ${ }^{(22)}$, que encontrou em seu trabalho valor de $0,5 \mathrm{ng} / \mathrm{mL}$ para a CKMB massa.

Em relação à CK-MB massa, sugere-se utilizar os valores achados neste estudo, ou seja, média: $0,9418 \mathrm{ng} / \mathrm{mL}$, com IC: $\pm 0,323(0,618-1,264 \mathrm{ng} / \mathrm{mL})$, para valores de referência em futuros trabalhos. Os valores encontrados foram diferentes dos registrados por Ferreira et al. ${ }^{(22)}$ e Santos et al. ${ }^{(2)}$, o que pode ter ocorrido por diversos fatores, como a quantidade de animais utilizados em cada trabalho. Na presente pesquisa, utilizaram-se $n=60$ animais, enquanto na de Santos et al. ${ }^{(2)}$ empregaram-se 38 animais, sendo somente 20 sadios. Por sua vez, no estudo de Ferreira et al. ${ }^{(22)}$ somente um animal foi utilizado. Outro fator que também pode ser considerado é a região em que foi realizada a pesquisa; o presente trabalho e o de Ferreira et al. ${ }^{(22)}$ foram realizados em Campos dos Goytacazes/RJ, enquanto o de Santos et al. ${ }^{(2)}$ foi realizado em São Paulo/SP.

Na Figura 3, pode-se observar uma comparação dos valores obtidos de CK-MB massa neste estudo, com os valores obtidos por Ferreira et al. ${ }^{(22)}$ e Santos et al. ${ }^{(2)}$. 


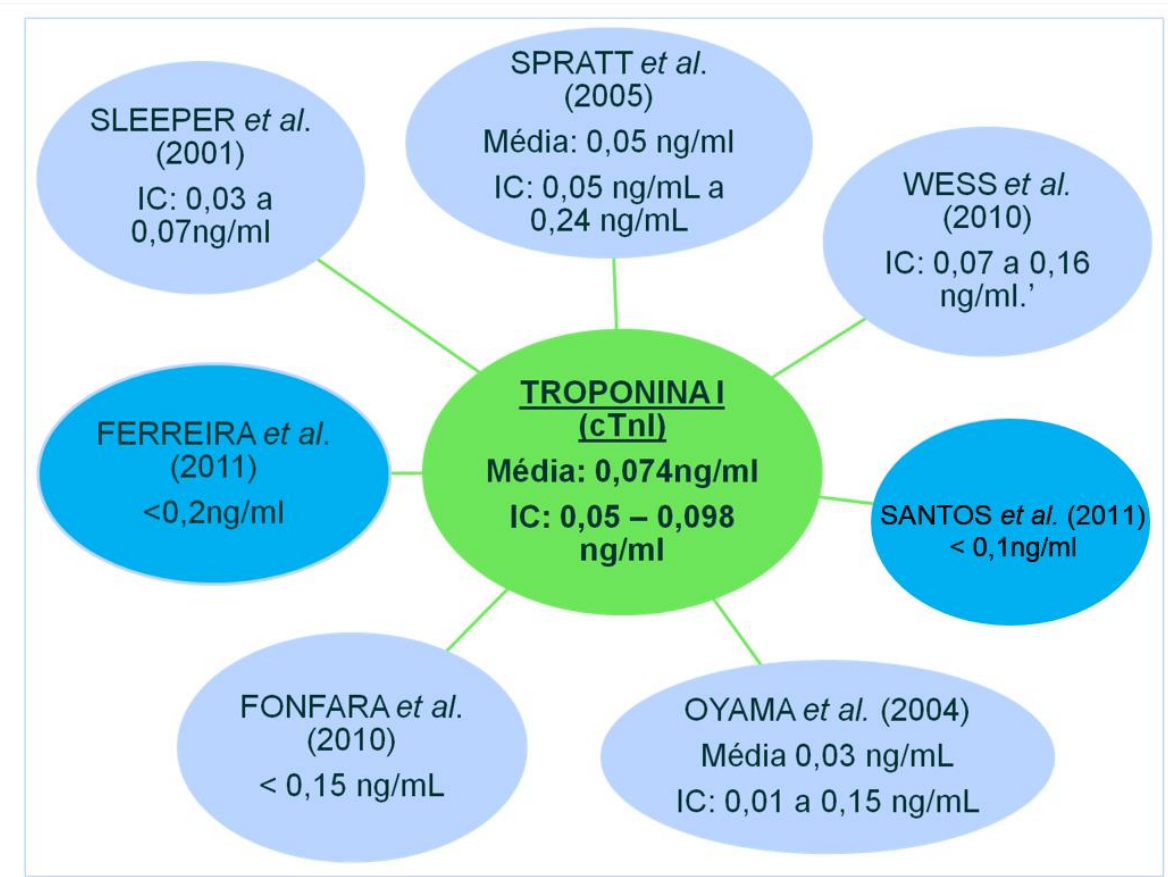

Figura 2 - Comparação dos resultados obtidos para a cTnI em relação aos valores disponíveis na literatura consultada.

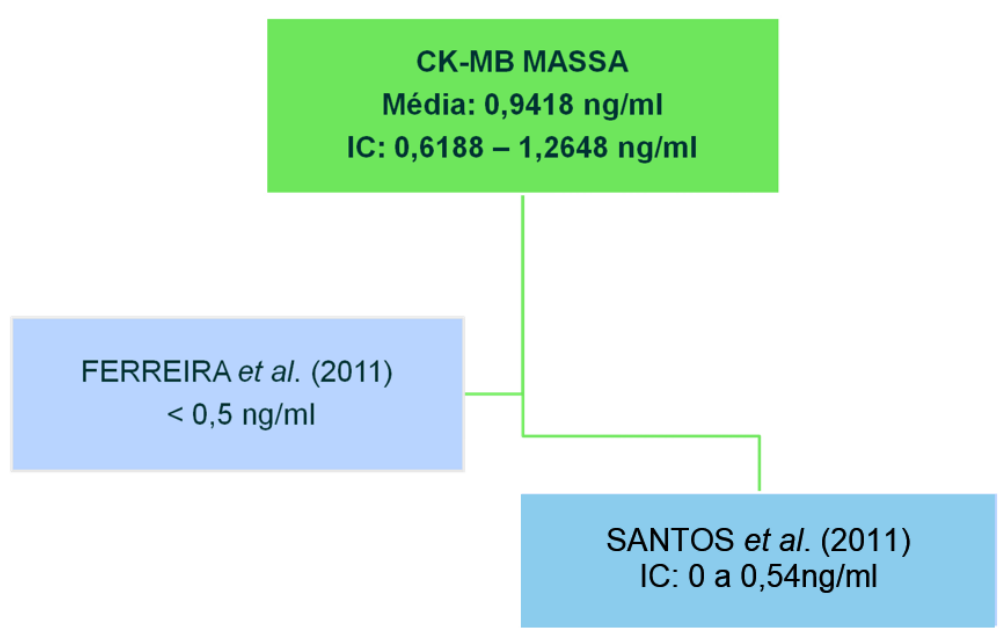

Figura 3: Comparação dos dados obtidos com os valores da literatura consultada, de CKMB Massa. Hospital Veterinário/UENF, 2012.

Como na presente pesquisa foram empregados cães comprovadamente hígidos, pode-se considerar os valores obtidos como referenciais. Além de saudáveis, o número de animais utilizados (60) foi consideravelmente maior do que foi o utilizado nos estudos de Ferreira et al. ${ }^{(22)}$ e Santos et al. ${ }^{(2)}$, o que aumenta a confiabilidade estatística para os valores aqui apresentados. 


\section{Conclusão}

Após análise dos resultados obtidos, concluiu-se que o kit de ensaio imunométrico por quimioluminescência turbo humano pode ser utilizado na espécie canina para a avaliação quantitativa da Troponina I e CK-MB massa, para o monitoramento de possíveis danos ao miocárdio na rotina clínica, como referência diagnóstica, ou em trabalhos experimentais.

\section{Agradecimentos}

Ao Laboratório Plínio Bacelar em Campos dos Goytacazes/RJ e ao Laboratório de Patologia Clínica da Universidade Estadual do Norte Fluminense (UENF), Campos dos Goytacazes/RJ.

\section{Referências}

1. Silva SH, Moresco RN. Biomarcadores cardíacos na avaliação da síndrome coronariana aguda. Scientia Medica. 2011;21(3):132-142.

2. Santos ALF, Larsson MHMA, Pereira GG, Santos MM, Gutierrez VCR. Dosagem sérica de troponina I em cães com desnível do segmento ST utilizando quimioluminescência. Arquivo Brasileiro de Medicina Veterinária e Zootecnia. 2011;63(6):1330-1336.

3. Freitas MV, Ferreira FS, Barretto FL, Baptista CB. Creatinofosfoquinase-isoenzima MB (CK-MB) e Troponina I (cTnI) em cães Revisão de Literatura. MEDVEP. Revista Cientifica de Medicina veterinária. Pequenos Animais e Animais de Estimação. 2014;11:156-165.

4. Lopes STA, Franciscato C, Teixeira LV, Oliveira TGM, Garmatz BC, Veiga APM, Mazzanti A. Determinação da Creatina Quinase em Cães. Revista da FZVA. 2005;12(1):116-122.

5. Diniz PPVP, Schwartz DS, Collicchio-Zuanaze RC. Cardiac trauma confirmed by cardiac markers in dogs: two case reports. Arquivo Brasileiro de Medicina Veterinária e Zootecnia. 2007;59(1):85-89.

6. Camplesi AC, Simao NMB, Sakate M, Sobreira MFR, Bersano PRO, Freitas SH, Moya-Araujo CF. Clinical and laboratory evaluation of dogs experimentally intoxicated with toad venom. Scientific Journal of Animal Science. 2013;2(11):323-333.

7. Aktas DM, Auguste D, Lefebvre HP, Toutain PL, Braun JP. Creatine kinase in the dog: a review. Veterinary Research Communications. 1993;17(5):353-369.

8. Yonezawa LA, Silveira VF, Machado LP, Kohayagawa A. Marcadores cardíacos na medicina veterinária. Ciência Rural. 2009;40(1):1-9.

9. Godoy MF, Braile DM, Purini Neto JA. Troponina como Marcador de Injúria Celular Miocárdica. Arquivo Brasileiro de Cardiologia. 1998;71(4):629-633.

10. Boswood A. Biomakers in cardiovascular disease: beyond natriuretic peptides. Journal of Veterinary Cardiology. 2009;11:23-32.

11. Kocaturk M, Martinez S, Eralp O, Tvarijonaviciute A, Ceron J, Yilmaz, Z. Tei index (myocardial performance index) and cardiac biomarkers in dogs with parvoviral enteritis. Research in Veterinary Science. 2012;92(1):24-29. 
12. Leal JCF, Braile DM, Godoy MF, Purini Neto J, Paula Neto A, Ramin SL, Zaiantchick M. Avaliação imediata da Troponina I cardíaca em pacientes submetidos à Revascularização do miocárdio. Revista Brasileira de Cirurgia Cardiovascular. 1999;14(3):247-253.

13. Sleeper MM, Clifford CA, Laster LL. Cardiac Troponin I in the Normal Dog and Cat. Journal of Veterinary Internal Medicine. 2001;15(5):501-503.

14. Amaral AS, Gaspar LFJ, Hennemann CRA. Valores de referência de constituintes bioquímicos séricos para cães da região de Santa Maria, RS. Revista da FZVA. 1996;3(1):86-97.

15. Adin DB, Milner RJ, Berger KD, Engel C, Salute M. Cardiac troponin I concentrations in normal dogs and cats using a bedside analyzer. Journal of Veterinary Cardiology. 2005;7(1):27-32.

16. Herndon WE, Kittleson MD, Sanderson K, Drobatz KJ, Clifford CA, Gelzer A, Summerfield NJ, Linde A, Sleeper MM. Cardiac Troponin I in feline hypertrophic cardiomyopathy. Journal Veterinary Internal Medicine. 2002;16(3):558-564.

17. Schober KE, Cornand C, Kirbach B, Aupperle H, Oechtering G. Serum cardiac troponin I and cardiac troponin $\mathrm{T}$ concentra- tions in dogs with gastric dilatation--volvulus. Journal of the American Veterinary Medical Association. 2002;221(3):381-388.

18. Spratt DP, Mellanby RJ, Drury N, Archer J. Cardiac troponin I: evaluation of a biomarker for the diagnosis of heart disease in the dog. Journal of Small Animal Practice. 2005;46(3):139-145.

19. Fonfara S, Loureiro J, Swift S, James R, Cripps P, Dukes-Mcewan J. Cardiac troponin I as a marker for severity and prognosis of cardiac disease in dogs. The Veterinary Journal. 2010;184(3):334-339.

20. Wess G, Simak J, Mahlink M, Hartmann K. Cardiac Troponin I in Doberman Pinschers with Cardiomyopathy. Journal of Veterinary Internal Medicine. 2010;24(4):843-849.

21. Oyama MA, Sisson DD. Cardiac troponin-I concentration in dogs with cardiac disease. Journal of Veterinary Internal Medicine. 2004;18(6):831-839.

22. Ferreira FS, Bustamante SB, Barreto FL, Correa ES, Freitas MV, Oliveira ALA, Carvalho CB. Troponina I cardíaca (cTnI) e CK-MB massa em cão submetido a circulação extracorpórea - Relato de caso. MEDVEP. Revista Cientifica de Medicina veterinária. Pequenos Animais e Animais de Estimação. 2011;8(27):572-578. 